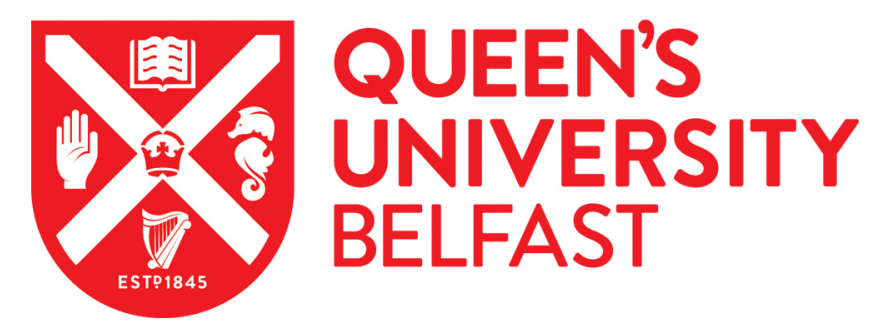

\title{
Quality of life in advanced renal disease managed either by haemodialysis or conservative care in older patients
}

McKeaveney, C., Witham, M., Alamrani, A. O. M., Maxwell, P., Mullan, R., Noble, H., Shields, J., \& Reid, J. (2020). Quality of life in advanced renal disease managed either by haemodialysis or conservative care in older patients. BMJ Supportive and Palliative Care. https://doi.org/10.1136/bmjspcare-2020-002237

Published in:

BMJ Supportive and Palliative Care

Document Version:

Peer reviewed version

Queen's University Belfast - Research Portal:

Link to publication record in Queen's University Belfast Research Portal

Publisher rights

Copyright 2020 BMJ

This manuscript is distributed under a Creative Commons Attribution-NonCommercial-NoDerivs License

(https://creativecommons.org/licenses/by-nc-nd/4.0/), which permits distribution and reproduction for non-commercial purposes, provided the author and source are cited.

\section{General rights}

Copyright for the publications made accessible via the Queen's University Belfast Research Portal is retained by the author(s) and / or other copyright owners and it is a condition of accessing these publications that users recognise and abide by the legal requirements associated with these rights.

Take down policy

The Research Portal is Queen's institutional repository that provides access to Queen's research output. Every effort has been made to ensure that content in the Research Portal does not infringe any person's rights, or applicable UK laws. If you discover content in the Research Portal that you believe breaches copyright or violates any law, please contact openaccess@qub.ac.uk. 
1 Quality of life in advanced renal disease managed either by haemodialysis or conservative care 2 in older patients.

4 Dr Clare McKeaveney

5 School of Nursing and Midwifery, Medical Biology Centre, Queen's University Belfast, Belfast, 6 UK.

Professor Miles Witham

NIHR Newcastle Biomedical Research Centre, Newcastle University, Newcastle upon Tyne, UK. 10

Miss Abrar Omran M Alamrani

12 School of Nursing and Midwifery, Medical Biology Centre, Queen's University Belfast, Belfast, 13 UK.

14

15

Professor Alexander P. Maxwell

16 Centre for Public Health, Queen's University Belfast, Institute of Clinical Science, Royal

17 Victoria Hospital, Grosvenor Road, Belfast, BT12 6BA, Northern Ireland. Regional Nephrology

18 Unit, Belfast City Hospital, Belfast Health Social Care Trust, Belfast, BT9 7AB, Northern

19 Ireland.

Dr Robert Mullan

Department of Nephrology, Antrim Area Hospital, Northern Health Social Care Trust, Antrim, BT41 2RL, Northern Ireland.

Dr Helen Noble, PhD, School of Nursing and Midwifery, Queen's University Belfast, UK,

Dr Joanne Shields

Regional Nephrology Unit, Belfast City Hospital, Belfast Health Social Care Trust, Belfast, BT9 $7 A B$, Northern Ireland.

Professor Joanne Reid*

School of Nursing and Midwifery, Medical Biology Centre, Queen's University Belfast, Belfast, UK.

*Corresponding author Professor Joanne Reid, School of Nursing and Midwifery, Queen's University Belfast, 97 Lisburn Road, Belfast, BT9 7BL, j.reid@qub.ac.uk, Tel: 0044289097 2459. 


\section{Abstract:}

2 Objective: Consideration of quality of life $(\mathrm{Q} O L)$ in people with end-stage renal disease (ESRD)

3 has become an important part of treatment decision-making. The aim of this study was to 4 report on QoL and other functional outcomes in patients with advanced chronic kidney disease 5 (CKD). Method: This was a cross-sectional study. Two samples of older patients ( $>60$ years old) 6 either conservatively managed (CM) or receiving hospital-based haemodialysis (HD), 7 compared kidney disease quality of life (KDQoL-36) outcomes. Results: Data from 263 CM patients (CKD $4 \mathrm{n}=188$, mean age 73.6 years, 48 females; CKD $5 n=75$, mean age 74.4 years, 26 female) and 74 patients on HD (mean age 73.8 years, 24 female) were analysed. Significant group differences were identified for two subscales of KDQoL-36. Symptoms/Problems List subscale was significantly better for those receiving HD compared to those CM with CKD 5 $(p=<.001)$. Symptom/Problem List scores of CM CKD stage 4 patients were not significantly different compared to HD patients but were significantly better than CM CKD stage 5 patients $(p<.001)$. Burden of Kidney Disease subscale was significantly better for both CKD $4(p<.001)$ and CKD 5 ( $p<.001)$ CM patients when compared to those receiving HD. Conclusion: Symptoms of advanced CKD significantly impact QoL for patients CM with CKD stage 5. Conversely, QoL is significantly impacted for those in receipt of haemodialysis due to the burden of treatment. These findings provide evidence for the use of QoL tools to help with clinical prognostication in advanced CKD. Utilising QoL tools will ensure specialist support is available for appropriate management of patients with CKD.

Key words: End-stage renal disease, conservative care, haemodialysis, quality of life

\section{Introduction}

23 End-stage renal disease (ESRD) is increasingly common, in part due to a rapid expansion in the numbers of older people, but also due to advances in the management of comorbidities (particularly cardiovascular disease) associated with chronic kidney disease (CKD). (1) Although renal replacement therapy (dialysis or renal transplantation) are well established interventions for ESRD, these treatments carry significant risks and impose a substantial burden of care on patients. These burdens may outweigh the benefits in some groups of patients with ESRD, particularly those with multi-morbidity due to advancing age. (2) 
1 A rapidly ageing and multi-morbid patient population has led to the default clinical practice of dialyzing older and sicker patients. There is a growing need to find non-dialytic alternatives for ESRD. $(1,3)$ Conservative management $(C M)$ is considered an alternative choice to dialysis for ESRD (2) and is provided by a multidisciplinary team often in liaison with the community team and general practitioner. Although only limited epidemiologic evidence exists, it has been suggested that as many as one in seven ESRD patients may opt not to dialyze. (4) Addressing these end-of-life issues is an important component of caring for persons with ESRD but there is still limited agreement on the optimal clinical tools for prognostication. $(4,5)$

9 To date, research suggests there is no survival advantage among patients aged $>80$ years choosing dialysis over conservative management (6), particularly for those with higher levels of comorbidities and poorer functional status. (7) A study conducted in the United States reported $>75$-year olds will account for over $25 \%$ of ESRD patients (8), which will carry increased morbidity and mortality issues (9) whereby dialysis may not be suitable in all cases.

14 Quality of life is an important domain consideration by health care professionals in such circumstances. Patients with ESRD commonly experience a compromised QoL and severe symptom burden (10) which can be amplified in older patient populations. It is reported that ESRD patients suffer significantly and have similar, if not greater, symptom severity than cancer populations. (11) Accurate projections about survival and expected QoL would help to guide appropriate patient decision-making in ESRD, where balancing the impact of therapies on lifespan, symptoms and quality of life is crucial. However, there continues to be limited evidence within renal disease.

A systematic review (12) examining quality of life of older patients with ESRD undergoing conservative management found three studies directly comparing $\operatorname{CM}$ and $\operatorname{HD}(13,14,15)$. Only one study used KDQoL-SF with CKD stage 5 patients receiving conservative management $(n=101)$ compared to those who commenced dialysis $(n=38)$. QoL was similar between both groups after two years (13) however, patients were all $>75$ years and/or had high comorbidity burden (Charlson Comorbidity Index score of >8). The SF-36 was used in the two remaining studies with mixed findings. De Biase et al. (14) reported similar quality of physical and mental outcomes for age-matched CKD stage 5 CM and HD patients. Da Silva-Gane et al. (15) reported poorer quality of physical health for the CM group whereas quality of mental health was similar 
1 between $\mathrm{CM}$ and those that transited to HD. Interestingly, life satisfaction reduced 2 significantly for those that initiated dialysis; which did improve at follow up. A recent cross3 sectional study (16) also conducted in the United States, used the KDQoL-36 reporting significant levels in Burden and Effect of Kidney Disease subscales in HD patients compared to those CM. However, the authors highlight heterogeneity between the groups concerning comorbid conditions. Overall, the current evidence base is limited and there is a need for further studies. $(12,16)$

\section{Materials \& Methods:}

\section{Aim}

10 The primary objective of this paper was to explore QoL findings in two contrasting studies of 11 older patients with advanced CKD. Study one included a group of CKD stage 5 patients receiving HD. Study two included a group of patients with advanced CKD (stage 4 \& stage 5) managed conservatively. Protocols for these studies are published elsewhere. $(17,18)$ Crosssectional QoL findings and functional outcomes were compared for HD and CM patients.

\section{Study population}

16 Study 1: Adult haemodialysis (HD) patients

17 Patients were recruited during haemodialysis (17). HD patients $>60$ years $(n=74)$ who attended

18 two haemodialysis units within the United Kingdom and had a confirmed diagnosis of ESRD

19 (estimated GFR $<15 \mathrm{~mL} / \mathrm{min} / 1.73 \mathrm{~m}^{2}$ ) were recruited. HD patients completed the Functional 20 Assessment of Chronic Illness Therapy (FACIT) questionnaire and handgrip strength (HGS) was 21 recorded. All patients were able to read and write English and were over 18 years of age. Data 22 were collected between September 2017 and April 2018.

23 Study 2: Conservative management (CM) patients

24 This study collected data on QoL in a CM patient group (18) recruited to a randomised controlled trial of oral bicarbonate therapy vs placebo. This study included CM patients $26(n=263)$ who attended 24 study sites within the United Kingdom aged $>60$ years, with an eGFR $27 \leq 30 \mathrm{~mL} / \mathrm{min} / 1.73 \mathrm{~m}^{2}$, with serum bicarbonate levels $<22 \mathrm{mmol} / \mathrm{L}$ and not on dialysis. This group 
1 includes CKD stage 4 who do not have ESRD, and so are not (yet) at a point where one would 2 have to make a choice about dialytic vs non-dialytic therapy. However, these patients are

3 under conservative management insofar as they are not being dialysed and may never need to be dialysed. In addition to QoL measures, the six-minute walk test (6MWT) was completed by patients and handgrip strength (HGS) was recorded. Data were collected between 2014 and 62016.

\section{Data collection}

$8 \mathrm{HD}$ and CM patients in each study were asked to complete Likert subscales of the Kidney 9 Disease Quality of Life (KDQoL-36 (HD) or the original 134-item KDQoL version (CM); 1) 10 Symptoms of kidney disease, 2) Effects of kidney disease, 3) Burden of kidney disease, 4) SF12 11 physical component score (PCS), 5) SF-12 mental component score (MCS). These sub-scales can be compared across the two tools, the appropriateness of which is outlined in the literature (19). The raw scores are transformed linearly ranging from 0 to 100 using an Excel scoring template. A higher score indicates better QoL. KDQoL-36 has confirmed reliability and validity in measuring QoL in an ESRD population (19) (reliability across subscales [a $=.81-.87$ ] and construct validity $[r=.4-.5])$. It has also been validated and translated into various languages $(20,21,22)$. HGS was measured in all patients. It is a reliable screening assessment for functional limitations and strongly correlates with other functional assessments such as 6MWT. (23) The 6MWT was recorded for CM patients whereas the HD patients completed the

20 Functional Assessment of Chronic Illness Therapy (FACIT) questionnaire; a measure of fatigue. (24) Additional patient information was also collated (e.g. marital status, ethnicity and comorbidities (yes/no for hypertension, myocardial infarction, peripheral vascular disease, 23 diabetes mellitus).

\section{Data analysis}

Patients were age matched. Descriptive statistics were generated for baseline demographics (sex, age) and QoL subscales. ANOVA was performed to compare KDQoL subscales between HD and CM (CKD stage 4 and CKD stage 5) populations. Bivariate correlations were conducted to assess any associations between KDQOL-36 subscales and functionality. Scale scores are computed with the KDQOL-36 ${ }^{\mathrm{TM}}$ scoring program. Participants with any missing data on the relevant measures were excluded from analysis $(n=22)$. The significance level for all statistical 
1 analyses was $p<.05$. All analyses were conducted using SPSS version 24 (IBM Corp. Released

2 2016, IBM SPSS, Statistics for Windows, Version 24.0, IBM Corp, Armonk, NY).

\section{Results:}

4 Data from 263 CM (CKD stage $4 \mathrm{n}=188$, mean age 73.6 years, 48 females; CKD stage $5 \mathrm{n}=75$, 5 mean age 74.4 years, 26 females) and 74 patients on HD (mean age 73.8 years, 24 female) were analysed (table 1). Mean scores for KDQoL subscales are presented in Table 2. Higher scores indicate better QoL. Symptom or problem list subscale was significantly different between groups $(F(2,334)=9.170 ; p<.001)$. HD patients had significantly better scores compared to CM CKD stage $5(p<.001)$. Scores for CM CKD stage 4 patients were also significantly better when compared to CM CKD stage $5(p<.001)$ only. There were no significant differences between patient groups for the Effects of Kidney Disease subscale $(F(2,334)=$ 2.414; $p=.091)$. The burden of kidney disease subscale was significantly different between patient groups $(F(2,334)=133.339 ; \mathrm{p}<.001)$. CM CKD stage $4(M=87.88 ; \mathrm{SD}=13.62)$ and $\mathrm{CKD}$ stage $5(M=82.80 ; S D=15.39)$ scores were not dissimilar but were significantly better than HD patients $(p<.001)$. There were no significant differences between patient groups for SF-12 Physical Component Subscale $(F(2,334)=.573 ; p=.565)$ or SF-12 Mental Component Subscale between patient groups $(F(2,334)=.317 ; \mathrm{p}=.302)$.

Mean scores between groups were not statistically dissimilar for Effects of Kidney Disease, SF12 PCS or SF-12 MCS. However, CM CKD stage 4 patients tended to have better KDQoL scores compared to HD and CM CKD stage 5 patients (Burden of Kidney Disease; SF-12 PCS, SF-12 MCS). CM individuals with CKD stage 5 also tended to have worse KDQoL scores (Symptoms/Problem List, Effect of Kidney Disease, SF-12 MCS). Bivariate analysis (Table 3) was conducted comparing associations between quality of life subscales and HGS for patient groups. Significant associations were reported for Burden of Kidney Disease and HGS in CM CKD stage $5(r=.233)$ and SF-12 PCS and HGS in HD patients $(r=.319)$.

Table 1: Patient characteristics 


\begin{tabular}{|l|l|l|l|}
\hline sex Male (\%) & $140(74 \%)$ & $49(65 \%)$ & $58(78 \%)$ \\
\hline Mean AGE years (SD) & $73.6(6.6)$ & $74.4(7.9)$ & $73.8(7.6)$ \\
\hline Ethnicity White (\%) & $184(98 \%)$ & $70(93 \%)$ & $74(100 \%)$ \\
\hline Living arrangements & $55(29 \%)$ & $23(33 \%)$ & $22(30 \%)$ \\
\hline Living alone (\%) & $164(89 \%)$ & $67(89 \%)$ & $60(81 \%)$ \\
\hline Co-morbidities & $32(17 \%)$ & $4(5 \%)$ & $11(15 \%)$ \\
\hline Hypertension & $16(9 \%)$ & $6(8 \%)$ & $24(31 \%)$ \\
\hline Myocardial infarction & $62(33 \%)$ & $22(29 \%)$ & $56(75 \%)$ \\
\hline Peripheral Vascular Disease & & & \\
\hline Diabetes mellitus & & & \\
\hline ^reference group for Anova & & & \\
\hline
\end{tabular}

2 Table 2: KDQOL-36 subscales

\begin{tabular}{|l|l|l|l|}
\hline KDQOL-36 subscales & CM CKD 4 (N=188) & CM CKD 5 (N=75) & $H D(n=74)^{\wedge}$ \\
\hline Symptom or problem list & $77.8(24.4)^{* *}$ & $66.5(25.2)^{*}$ & $81.3(13.7)$ \\
\hline Effects of kidney disease & $80.9(12.5)$ & $79.0(13.4)$ & $83.8(15.2)$ \\
\hline Burden of kidney disease & $87.9(13.6)^{*}$ & $82.8(15.4)^{*}$ & $47.0(29.2)$ \\
\hline SF-12 PCS & $38.8(11.3)$ & $37.7(8.9)$ & $37.5(9.8)$ \\
\hline SF-12 MCS & $51.9(10.6)$ & $49.8(9.6)$ & $51.4(9.3)$ \\
\hline${ }^{\wedge}$ reference group for ANOVA; * ${ }^{*}<.001, \sigma^{*}$ respective post-hoct-test of CM CKD 4 vs. CM CKD 5
\end{tabular}

3 Table 3: Bivariate correlations between quality of life subscales and measures of functionality

4 for CM (CKD stage 4 \& 5) and HD

\begin{tabular}{|c|c|c|c|c|}
\hline KDQoL subscales & & CM CKD $4(\mathrm{~N}=188)$ & CM CKD $5(\mathrm{~N}=75)$ & $\mathrm{HD}(\mathrm{N}=74)$ \\
\hline Symptoms/problem list & \multirow{3}{*}{$\begin{array}{c}\text { HGS } \\
\text { (higher } \\
\text { score }=\end{array}$} & .096 & .174 & .217 \\
\hline Effects of Kidney Disease & & -.018 & .153 & .085 \\
\hline Burden of Kidney Disease & & 117 & $.233^{*}$ & .064 \\
\hline
\end{tabular}




\begin{tabular}{|c|c|c|c|c|}
\hline SF-12 PCS & \multirow{2}{*}{$\begin{array}{c}\text { higher } \\
\text { function) }\end{array}$} & -.135 & -.011 & $.319 *$ \\
\hline SF-12 MCS & & .063 & .140 & -.029 \\
\hline Symptoms/problem list & \multirow{5}{*}{$\begin{array}{l}\text { 6MWT } \\
\text { (higher } \\
\text { score = } \\
\text { greater } \\
\text { distance } \\
\text { covered) }\end{array}$} & .108 & .038 & - \\
\hline Effects of Kidney Disease & & $.263^{* *}$ & .202 & - \\
\hline Burden of Kidney Disease & & $.234^{* *}$ & .108 & - \\
\hline SF-12 PCS & & $.385^{* *}$ & $.251^{*}$ & - \\
\hline SF-12 MCS & & $.158^{*}$ & .218 & - \\
\hline Symptoms/problem list & \multirow{5}{*}{$\begin{array}{c}\text { FACIT } \\
\text { (higher } \\
\text { score }= \\
\text { lower } \\
\text { fatigue) }\end{array}$} & - & - & $.628^{* *}$ \\
\hline Effects of Kidney Disease & & - & - & $.374^{* *}$ \\
\hline Burden of Kidney Disease & & - & - & $.357^{* *}$ \\
\hline SF-12 PCS & & - & - & $.540 * *$ \\
\hline SF-12 MCS & & - & - & $.557^{* *}$ \\
\hline
\end{tabular}

$\left({ }^{*} p=<.05 ;{ }^{* *} p=<.001\right)$ HGS: handgrip strength; 6MWT: six-minute walk test; FACIT: Functional Assessment of Chronic Illness Therapy

2 Table 3 provides positive correlations between KDQoL-36 subscales and HGS. Although

3 significant, weak correlations between Burden of Kidney Disease and HGS $(r=.233, p=.044)$ in

4 CM CKD 5, suggests better scores on Burden of Kidney Disease are associated with higher HGS.

5 A significant but weak correlation between SF-12 PCS and HGS in HD patients $(r=.319, p=.006)$

6 suggests increases in SF-12 PCS are associated with higher HGS. The 6MWT was administrated

7 only to CM patients. With exception of Symptoms/Problems List subscale, higher scores of

8 Effects $(p<.001)$, Burden ( $p=.001)$, SF-12 PCS $(p<.001)$ and SF-12 MCS ( $p=.031)$ were associated

9 with longer walking distances in the 6MWT for CM CKD stage 4 patients. Correlations were of

10 a similar magnitude for CKD stage 5 but did not reach significance in all cases due to the smaller

11 sample size with exception of SF-12 PCS ( $r=.251 ; p=.031)$. Positive moderate correlations were

12 reported for all KDQoL subscales (Symptoms ( $p<.001)$, Effect $(p=.001)$, Burden $(p=.002)$, PCS

$13(p<.001), \operatorname{MCS}(p<.001))$ and FACIT $(p<.001)$ in HD patients. This suggests better KDQoL is

14 associated with better scores of fatigue. 


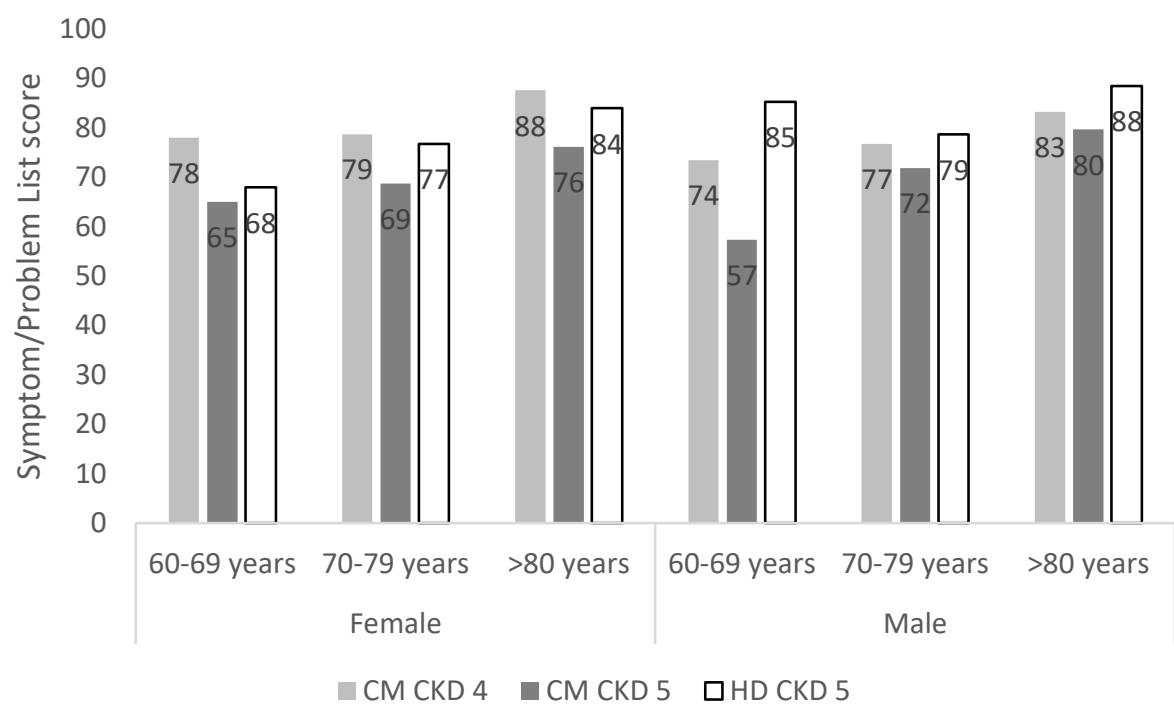

2 Figure 1 demonstrates the lower age group (60-69 years) tended to experience greater

3 symptoms or problems in both males and females. In addition, as age increased, QoL

4 symptom and problem list scores improve.

5 Insert [Fig 2: QoL Effects of Kidney Disease subscale for CM and HD patients by age and sex]

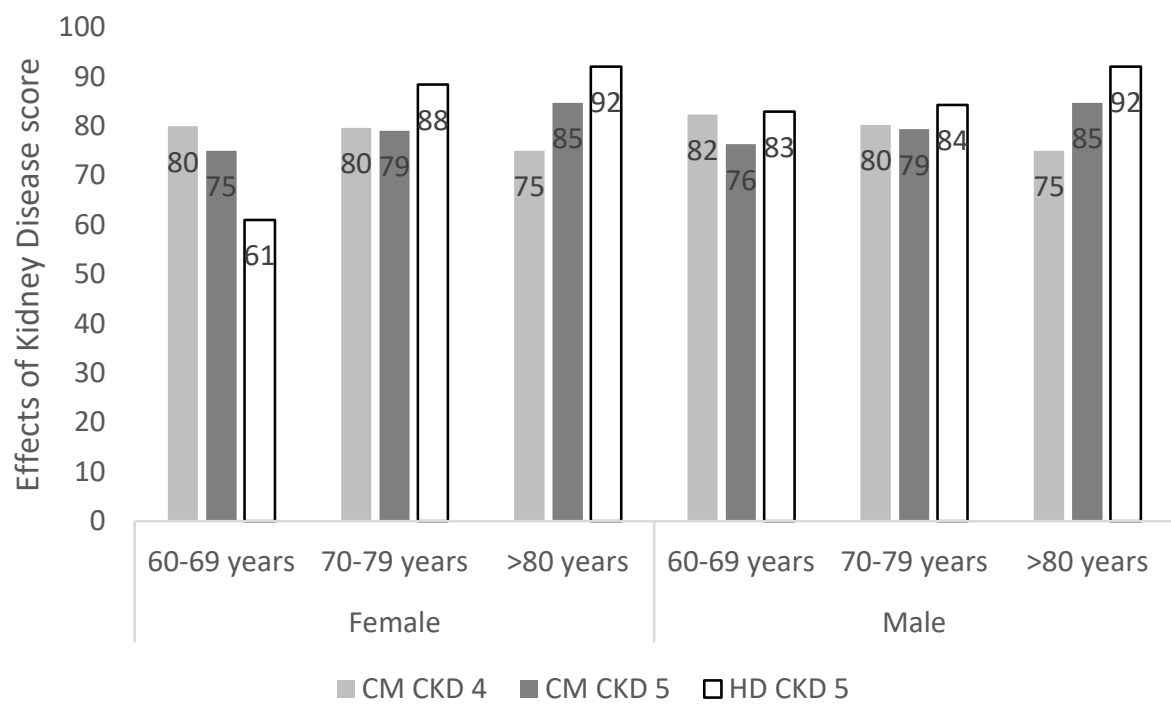

6

7

8 Figure 2 demonstrates higher scores were more common in HD groups for both males and 9 females.

10 Insert [Fig 3: QoL Burden of Kidney Disease subscale for CM and HD patients by age and sex] 


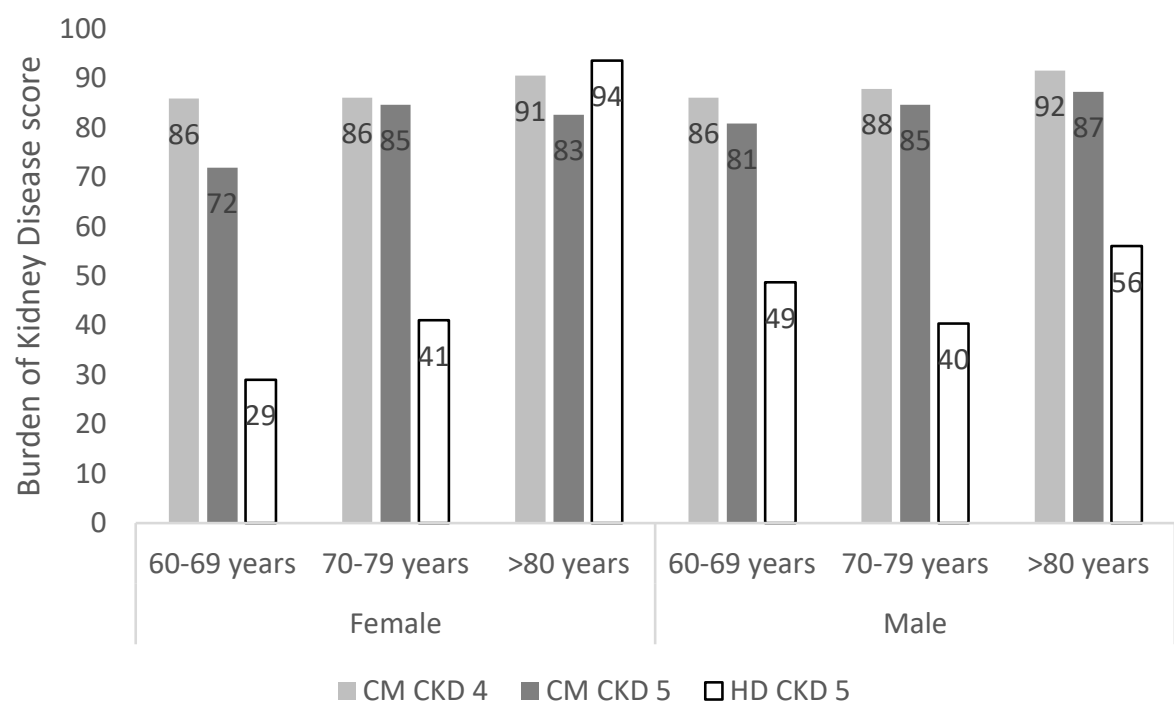

2 Figure 3 demonstrates males experiences less Burden of Kidney Disease compared to

3 females. HD patients experienced greatest Burden of Kidney Disease across age groups with 4 exception of females $>80$ years, indicated by lowest QoL scores.

Insert [Fig 4: QoL SF-12 Physical Component Subscale for CM and HD patients by age and sex]

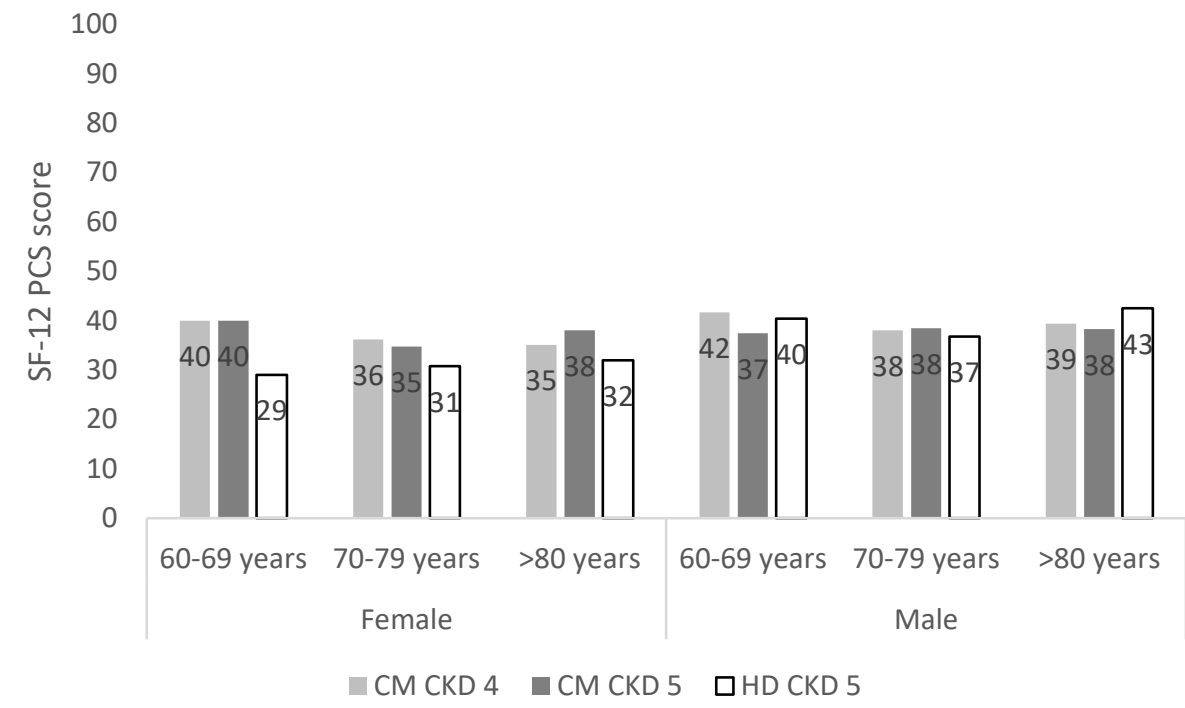

7 Figure 4 demonstrates males patients had better scores of SF-12 PCS compared to females.

8 Males tended to have similar scores across age groups.

9 Insert [Fig 5: QoL SF-12 Mental Component Subscale for CM and HD patients by age and sex] 


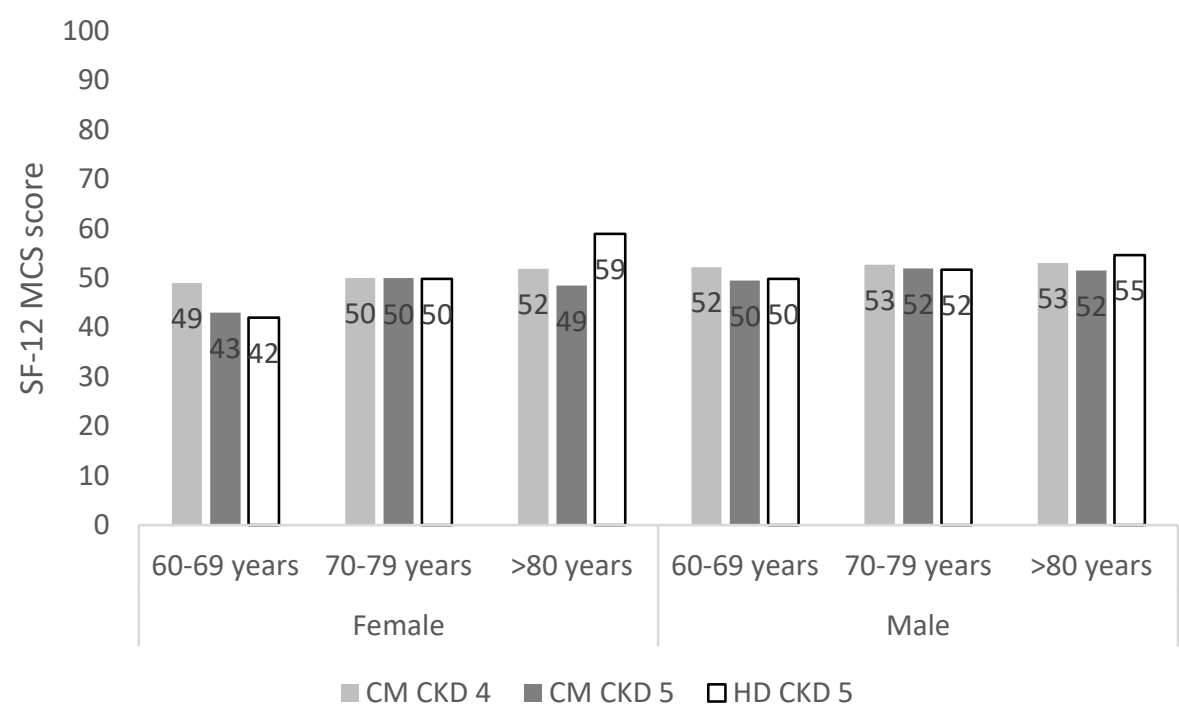

1

2 Figure 5 demonstrates males patients had better scores of SF-12 MCS compared to females.

3 Males tended to have similar scores across age groups. Females in the lower age group (60-

469 years) had the poorest quality of life for mental health.

\section{Discussion}

6 Few studies have examined QoL outcomes between patients opting for CM and HD treatment.

7 This study directly compares QoL between age-matched CM and HD populations using the 8 KDQoL-36 taking account of the CKD stage. In the current study, CM patients with CKD stage

95 tended to have poorer mean scores across more of the KDQoL subscales. This was

10 significantly lower for the Symptom/Problems List subscale when compared to CM CKD stage

114 and HD patients. Conversely, it has been suggested that KDQoL-36 is an insensitive measure

12 for HD patients (25) which includes a symptom and burden list that may be less prevalent since

13 its publication. This may help to explain findings in the current study. Ceiling effects have been

14 reported within other studies. (25) Severity of symptoms continues to be under recognised in

15 ESRD (26) and further work is required to identify key symptoms and the impact on patient's

16 quality of life.

17 Effects of Kidney Disease scores were not dissimilar between groups. CM CKD stage 5 patients

18 had the poorest scores whereas HD patients had better scores. The Effects of Kidney Disease

19 questions relate to a wide range of issues such as fluid restriction and dietary limitation,

20 financial hardship, impact of sexual dysfunction, work status and being dependent on medical 
1 personnel. (27) Answers to these issues are likely to be influenced by other patient factors such as current functionality and support needs. It is essential to explore these aspects.

3 Similar to Seow et al. (13) the Burden of Kidney Disease subscale was significantly poorer in HD patients compared to those receiving CM (CKD 4 and 5) in the current study. The nature of dialysis treatment is more likely to burden daily life as it includes invasive interventions, medicalisation and a significant time commitment required for dialysis therapies. (14) Also, post-dialysis treatment is associated with a wide range of adverse effects. (28)

The physical (PCS) and mental component (MCS) in HD and CM patients reported similar scores across groups. PCS and MCS scores were better for those managed conservatively with less severe renal disease i.e. CKD 4. As CKD progresses, patients typically have more comorbidities and have a higher mortality. ESRD patients experience a continual functional decline over time as well as an increasing risk of hospitalisation and premature death. (29-31) Monitoring physical function longitudinally (e.g. PCS scores) can be important assessment measures. However, Hall and colleagues (29) suggest that SF-12 PCS changes may not be meaningful for older dialysis patients. Objective physical performance measures (e.g. 6MWT) are more sensitive to subtle changes in physical function and therefore, may be more useful in tracking

17 functional decline.

CM CKD stage 5 patients reported lower MCS suggesting poorer mental health outcomes. Although scores $<50$ indicate poorer health related quality of life (HRQoL; 32), overall scores of MCS were not dissimilar to 'national norms' reported by Peipert and colleagues. (19) However, these scores refer to dialyzed patients. Further work is required to provide normative values for non-dialysis patients. In addition, it has been suggested that the PCS and MCS may not appropriate measures for CM CKD patients, as these do not include domains which become increasingly important at the end of life (such as existential concerns, comfort and peace of mind). (32) Global assessments such as the Integrated Palliative Care Outcome Scale (33), used alongside HRQoL measures, have been recommended. (32)

27 There is an undisputed strong relationship between physical functional assessments such as HGS and ability to perform daily living. (30) Assessment of functionality in this study has also helped to reinforce this finding. Better QoL was associated with stronger HGS in CM CKD stage 
15 and HD, longer walking distance in CM CKD stage 4 and more energy in HD patients. The 2 ability to engage in activities of daily living should be used in combination with other 3 assessments to understand the likely impact on QoL. KDQoL subscale scores also were similar across sex and different ages however females in the lower age group (60-69 years) tended to have poorer QoL scores. This is in keeping with QoL trends. For example, during the "third age" of life (65-85 years), QoL is reported to improve. In addition, women tend to have poor health related QoL. (34) With a growing older patient population, further research is needed to understand how QoL changes with increasing age and co-morbidity.

\section{Strengths and limitations}

10 This is a cross-sectional data and therefore cannot infer causality. CM tends to be offered more 11 frequently to older patients than dialysis in part due to greater perceived frailty and 12 comorbidities (35) however this study did not control for this. This study was also not adjusted 13 for sex and co-morbidities. Also, CKD 4 patients have been included who do not have end-stage

14 renal failure however, there is merit in including people with CKD 4 in our comparisons, in part because it demonstrates the relationship of severity of renal impairment with QoL. Other limitations include a lack of ethnic diversity which may influence findings as ethnic minorities tend to report lower QoL than white patient populations in self-rated health surveys. (36) Also, it is important to acknowledge the likely variability in the conservative management service provision between institutions which requires further exploration. Caution should be applied in the comparison of samples from two different studies conducted at different times, involving different demographic sites, however this work contributes to a small but growing evidence base investigating utility of QoL in older patients with ESRD.

\section{Implications of clinical practice}

24 Testing the utility of QoL clinical tools for prognostication is important; however, there is a need to update current KDQoL assessments (particularly symptom-related) taking account of evolving ESRD treatment (25). In addition, a prospective multicentre, ethnically diverse study overtime is required to provide a stronger evidence base for patient and clinician decision making. 


\section{Conclusion}

2 QoL for CM patients was better for those patients with less advanced kidney disease (CKD stage

34 vs. CKD stage 5). CM CKD 4 patients tended to have better QoL scores on more subscales

4 than HD or CM CKD 5. HD patients experienced a significantly greater burden of disease state

5 and treatment compared to the other groups. However, admission rates, complications and

6 other comorbidities require further exploration within these groups. (37) All groups

7 demonstrated low mental health scores highlighting a greater need for supportive care across

8 ESRD. Despite the challenges of studying QoL in this frail ESRD population, it is important to

9 determine what matters to individuals with ESRD. (35-37) Currently, this study highlights the

10 urgent need to provide enhanced support and services for patients conservatively managed.

\section{Funding}

12 Study 1 was funded by the Public Health Agency [STL/5179/15] and the Northern Ireland

13 Kidney Research Fund. Study 2 was funded by the National Institute for Health Research (NIHR)

14 Health Technology Assessment programme (project reference 10/71/01). The views expressed

15 are those of the authors and not necessarily those of the NIHR or the Department of Health

16 and Social Care.

17 Competing interests

18 No conflict of interest has been declared by the author(s).

19 Acknowledgements

20 Thanks are given to service users who assisted in this project. MDW acknowledges support 21 from the NIHR Newcastle Biomedical Research Centre.

\section{Authors' contributions}

$23 \mathrm{JR}$ is the principal investigator of the HD study and MDW is the principal investigator of the

24 CMK study. All authors have assisted in the design of the study and have revised and given 25 approval for the final version of the protocol. CMK completed data analysis. CMK, JR and MDW 
1 completed the initial draft of this manuscript. All authors read and approved the final 2 manuscript.

\section{Ethical approval}

4 Governance approval for the HD study was obtained from the Office of Research Ethics 5 Committees Northern Ireland (ORECNI; HD study 1- REC reference: 16/NI/0233) and was 6 funded by the Northern Ireland Public Health Agency / Northern Ireland Kidney Research Fund.

7 The BicARB trial, from which the $\mathrm{CM}$ data were obtained, was approved by the East of Scotland 8 NHS research ethics committee (approval 12/ES/0023), the Medicines and Healthcare 9 Regulatory Authority (EudraCT number 2011-005271-16; Clinical Trial Authorisation number 10 41692/0001/001-0001), and was funded by the National Institute for Health Research Health 11 Technology Assessment programme (reference 10/71/01).

12

13 Data availability statement

14 Data are available upon reasonable request. 


\section{References}

1. O'Connor NR, Kumar P. Conservative management of end-stage renal disease without dialysis: a systematic review. Palliat Med. 2012 Feb 1;15(2):228-35.

2. Thorsteinsdottir B, Swetz KM, Albright RC. The ethics of chronic dialysis for the older patient: time to reevaluate the norms. Clin J Am Soc Nephrol. 2015 Nov 6;10(11):20949.

3. Morton RL, Snelling P, Webster AC, Rose J, Masterson R, Johnson DW, Howard K. Factors influencing patient choice of dialysis versus conservative care to treat endstage kidney disease. CMAJ. 2012 Mar 20;184(5):e277-83.

4. Forzley B, Er L, Chiu HH, Djurdjev O, Martinusen D, Carson RC, Hargrove G, Levin A, Karim M. External validation and clinical utility of a prediction model for 6-month mortality in patients undergoing hemodialysis for end-stage kidney disease. Palliat Med. 2018 Feb;32(2):395-403.

5. Cohen LM, Ruthazer R, Moss AH, Germain MJ. Predicting six-month mortality for patients who are on maintenance hemodialysis. Clin J Am Soc Nephrol. 2010 Jan $1 ; 5(1): 72-9$.

6. Verberne WR, Geers AT, Jellema WT, Vincent HH, van Delden JJ, Bos WJ. Comparative survival among older adults with advanced kidney disease managed conservatively versus with dialysis. Clin J Am Soc Nephrol. 2016 Apr 7;11(4):633-40.

7. Murtagh FE, Burns A, Moranne O, Morton RL, Naicker S. Supportive care: comprehensive conservative care in end-stage kidney disease. Clin J Am Soc Nephrol 2016 Oct 7;11(10):1909-14.

8. Balogun RA, Turgut F, Balogun SA, Holroyd S, Abdel-Rahman EM. Screening for depression in elderly hemodialysis patients. Nephron Clin Pract. 2011;118(2):c72-7.

9. Lamping DL, Constantinovici N, Roderick P, Normand C, Henderson L, Harris S, Brown E, Gruen R, Victor C. Clinical outcomes, quality of life, and costs in the North Thames Dialysis Study of elderly people on dialysis: a prospective cohort study. Lancet. 2000 Nov 4;356(9241):1543-50.

10. Yong DS, Kwok AO, Wong DM, Suen MH, Chen WT, Tse DM. Symptom burden and quality of life in end-stage renal disease: a study of 179 patients on dialysis and palliative care. Palliat Med. 2009 Mar;23(2):111-9. 
11. Murtagh FE, Addington-Hall J, Edmonds P, Donohoe P, Carey I, Jenkins K, Higginson IJ. Symptoms in the month before death for stage 5 chronic kidney disease patients managed without dialysis. J Pain Symptom Manage. 2010 Sep 1;40(3):342-52.

12. Ren $Q$, Shi $Q$, Ma T, Wang J, Li Q, Li X. Quality of life, symptoms, and sleep quality of elderly with end-stage renal disease receiving conservative management: a systematic review. Health Qual Life Outcomes. 2019 Dec;17(1):1-9.

13. Seow YY, Cheung YB, Qu LM, Yee AC. Trajectory of quality of life for poor prognosis stage 5D chronic kidney disease with and without dialysis. Am J Nephrol. 2013;37(3):231-8.

14. De Biase V, Tobaldini O, Boaretti C, Abaterusso C, Pertica N, Loschiavo C, Trabucco G, Lupo A, Gambaro G. Prolonged conservative treatment for frail elderly patients with end-stage renal disease: the Verona experience. Nephrol Dial Transpl. 2008 Apr 1;23(4):1313-7.

15. Da Silva-Gane M, Wellsted D, Greenshields H, Norton S, Chandna SM, Farrington K. Quality of life and survival in patients with advanced kidney failure managed conservatively or by dialysis. Clin J Am Soc Nephrol. 2012 Dec 1;7(12):2002-9.

16. Shah KK, Murtagh FE, McGeechan K, Crail S, Burns A, Tran AD, Morton RL. Healthrelated quality of life and well-being in people over 75 years of age with end-stage kidney disease managed with dialysis or comprehensive conservative care: a crosssectional study in the UK and Australia. BMJ Open. 2019 May 17;9(5):e027776.

17. Reid J, Noble HR, Adamson G, Davenport A, Farrington K, Fouque D, Kalantar-Zadeh K, Mallett J, McKeaveney C, Porter S, Seres DS. Establishing a clinical phenotype for cachexia in end stage kidney disease-study protocol. BMC Nephrol. 2018 Dec $1 ; 19(1): 38$

18. Witham MD, Band MM, Littleford RC, Avenell A, Soiza RL, McMurdo ME, Sumukadas D, Ogston SA, Lamb EJ, Hampson G, McNamee P. Does oral sodium bicarbonate therapy improve function and quality of life in older patients with chronic kidney disease and low-grade acidosis (the BiCARB trial)? Study protocol for a randomized controlled trial. Trials. 2015 Dec 1;16(1):326.

19. Peipert JD, Bentler PM, Klicko K, Hays RD. Psychometric properties of the kidney disease quality of life 36-item short-form survey (KDQOL-36) in the United States. Am J Kidney Dis. 2018 Apr 1;71(4):461-8. 
20. Chow SK, Tam BM. Is the kidney disease quality of life-36 (KDQOL-36) a valid instrument for Chinese dialysis patients?. BMC Nephrol. 2014 Dec 1;15(1):199.

21. Ricardo AC, Hacker E, Lora CM, Ackerson L, DeSalvo KB, Go A, Kusek JW, Nessel L, Ojo A, Townsend RR, Xie D. Validation of the kidney disease quality of life short form 36 (KDQOL-36 $6^{\mathrm{TM}}$ ) US Spanish and English versions in a cohort of Hispanics with chronic kidney disease. Ethn Dis. 2013;23(2):202.

22. Mateti UV, Nagappa AN, Attur RP, Nagaraju SP, Mayya SS, Balkrishnan R. Cross-cultural adaptation, validation and reliability of the South Indian (Kannada) version of the Kidney Disease and Quality of Life (KDQOL-36) instrument. Saudi J Kidney Dis Transpl. 2015 Nov 1;26(6):1246.

23. Braun AK, Hess ME, Ibarra-Moreno U, Salvatore MD, Saunders NW. Handgrip strength as a screening assessment for functional limitations. Age. 2018;62(14.3):64-7.

24. Hays RD, Kallich JD, Mapes DL, Coons SJ, Carter WB. Development of the kidney disease quality of life (KDQOL) instrument. Qual Life Res. 1994 Oct 1;3(5):329-38.

25. Cohen E, Margalit I, Shochat T, Goldberg E, Krause I. The relationship between the concentration of plasma homocysteine and chronic kidney disease: a cross sectional study of a large cohort. J Nephrol. 2019 Oct 1;32(5):783-9.

26. Germain MJ, Davison SN, Moss AH. When enough is enough: the nephrologist's responsibility in ordering dialysis treatments. Am J Kidney Dis. 2011 Jul 1;58(1):135-43.

27. Gonçalves FA, Dalosso IF, Borba JM, Bucaneve J, Valerio NM, Okamoto CT, Bucharles SG. Quality of life in chronic renal patients on hemodialysis or peritoneal dialysis: a comparative study in a referral service of Curitiba-PR. J. Bras. Nefrol. 2015 Dec 1;37(4):467-74.

28. Eckert K, Motemaden L, Alves M. Effect of hemodialysis compared with conservative management on quality of life in older adults with end-stage renal disease: systematic review. J Hosp Palliat Nurs. 2018 Jun 1;20(3):279-85.

29. Hall RK, Luciano A, Pieper C, Colón-Emeric CS. Association of Kidney Disease Quality of Life (KDQOL-36) with mortality and hospitalization in older adults receiving hemodialysis. BMC Nephrol. 2018 Dec 1;19(1):11.

30. Porter AC, Lash JP, Xie D, Pan Q, DeLuca J, Kanthety R, Kusek JW, Lora CM, Nessel L, Ricardo AC, Nunes JW. Predictors and outcomes of health-related quality of life in adults with CKD. Clin J Am Soc Nephrol. 2016 Jul 7;11(7):1154-62. 
31. Seidel UK, Gronewold J, Volsek M, Todica O, Kribben A, Bruck H, Hermann DM. Physical, cognitive and emotional factors contributing to quality of life, functional health and participation in community dwelling in chronic kidney disease. PloS One. 2014 Mar 10;9(3):e91176.

32. Erez G, Selman L, Murtagh FE. Measuring health-related quality of life in patients with conservatively managed stage 5 chronic kidney disease: limitations of the Medical Outcomes Study Short Form 36: SF-36. Qual Life Res. 2016 Nov 1;25(11):2799-809.

33. Murtagh FE, Ramsenthaler C, Firth A, Groeneveld El, Lovell N, Simon ST, Denzel J, Guo P, Bernhardt F, Schildmann E, van Oorschot B. A brief, patient-and proxy-reported outcome measure in advanced illness: Validity, reliability and responsiveness of the Integrated Palliative care Outcome Scale (IPOS). Palliat Med. 2019 Sep;33(8):1045-57.

34. Lee $\mathrm{KH}, \mathrm{Xu} \mathrm{H}, \mathrm{Wu} \mathrm{B}$. Gender differences in quality of life among community-dwelling older adults in low-and middle-income countries: results from the Study on global AGEing and adult health (SAGE). BMC Public Health. 2020 Dec 1;20(1):114.

35. Tonkin-Crine S, Okamoto I, Leydon GM, Murtagh FE, Farrington K, Caskey F, Rayner H, Roderick P. Understanding by older patients of dialysis and conservative management for chronic kidney failure. Am J Kidney Dis. 2015 Mar 1;65(3):443-50.

36. Evandrou M, Falkingham J, Feng Z, Vlachantoni A. Ethnic inequalities in limiting health and self-reported health in later life revisited. J Epidemiol Community Health. 2016 Jul 1;70(7):653-62.

37. van Loon IN, Goto NA, Boereboom FTJ, Verhaar MC, Bots ML, Hamaker ME. Quality of life after the initiation of dialysis or maximal conservative management in elderly patients: a longitudinal analysis of the Geriatric assessment in OLder patients starting Dialysis (GOLD) study. BMC Nephrol. 2019 Mar 29;20(1):108. 